\title{
CHAPTER I: INTRODUCTION
}

\subsection{Background}

Potato (Solanum tuberosum L.) was initially introduced into Europe from South America around 1565 . Within 150 years of its introduction, potato became a staple in many European countries. Today, potato is fourth in total world food production and is produced throughout Europe, North and South America, Africa, Asia, Australia, and the Middle East (Daly 1996).

Potato has been successful due primarily to high yield per unit of land, as well as palatability, nutritional value, and ease of preparation. Although generally high yielding, many commercial potato cultivars are susceptible to a wide variety of pests including insects [Colorado potato beetle (Leptinotarsa decemlineata Say)], leafhoppers (Empoasca fabae Harris), aphids (Myzus persicae Sulzer and Macrosiphum euphorbiae Thomas), fungi [late blight (Phytophthora infestans de Bary)], bacterial wilts (Rhizoctonia), various nematodes [potato cyst nematode (Globodera pallida Stone and Globodera rostochiensis Wollenweber)], and viruses (PVY, PVX, PLRV). In addition, most cultivars are adapted to cool moist environments and do not perform well in semi-arid and sub-tropical environments.

Although there are 235 tuber-bearing species in the Solanaceae, only $S$. tuberosum subsp. tuberosum L. is panglobally cultivated with six other edible cultivated species primarily produced in Peru, Bolivia, and neighboring countries. Of the six cultivated species, S. phureja Juz. et Buk., S. stentomum Juz. et Buk., and S. ajanhuiri Juz. et Buk. are diploid $(2 n=2 x=24) ; S$. chaucha Juz. et Buk. and $S$. juzepczukii Buk. are triploid $(2 n=3 x=36)$; S. tuberosum subsp. tuberosum L. and subsp. andigena Hawkes are tetraploid $(2 n=4 x=48)$; and $S$. curtilobum Juz. et. Buk. is pentaploid $(2 n=5 x=60)$. The natural range of tuber bearing solanaceous species extends 
from the Southwestern United States through Mexico, Guatemala, Honduras, Costa Rica, and Panama and in every South American country except the Guianas. The tremendous ecological and geographical range of potato and its wild relatives is indicative of the morphological and physiological diversity found among the tuber-bearing Solanaceae. In addition, resistance genes have been found in many species for a broad array of fungal, bacterial, viral, insect, arachnid, and nematode pests. Thus, wild and diploid cultivated species of potato are a rich germplasm source providing opportunity for extensive improvement of European potato cultivars (Hawkes 1990).

\subsection{Obstructions to Genetic Gain}

S. tuberosum is a highly heterozygous crop which experiences severe inbreeding depression. Therefore, introgression of desirable traits from wild germplasm into a productive agronomic cultivar through repeated backcrossing is not possible. Since vigorous homozygous lines cannot be produced through conventional means, potato has primarily been vegetatively propagated, compounding problems with viral and other pathogenic infections. The autotetraploid nature of its genome complicates crossing, plant selection, genetic studies, and mapping of traits. Therefore, several breeding schemes have been proposed which utilize haploids to circumvent these barriers.

As used in this paper, haploids are plants with the gametic chromosome number, regardless of the parental chromosome number. Dihaploids are haploids derived from tetraploids, whereas doubled haploids derive from monoploid plants for which the chromosome number has doubled spontaneously or was induced to double through chemical application or tissue culture. Monoploids are a specific class of haploid plants possessing the basic 
chromosome number of the species.

\subsection{Haploid Breeding Schemes}

The first analytical haploid breeding scheme specific for potato was developed by Chase in 1963. He suggested that tetraploid clones be reduced through gynogenesis to the dihaploid level prior to breeding and selection. Once desirable diploid genotypes are obtained, they are crossed sexually to similar clones, resulting in progeny with low heterosis. The diploid progeny are doubled to the tetraploid level and resulting tetraploid lines are crossed to dissimilar clones yielding highly heterotic tetraploid cultivars analogous to double-cross hybrids of maize. Alternatively, chromosome doubling can be avoided by crossing dissimilar diploid progeny exhibiting low heterosis and a high frequency of unreduced gametes. The outcomes of the two methods are theoretically similar; however, somaclonal variation is a fairly common result of induced ploidy changes. In addition, the utilization of unreduced gametes eliminates a timeconsuming step (Chase 1963).

A second potato breeding scheme utilizing haploids was proposed by Wenzel et al. (1979) (Figure 1.1). The Analytical Synthetic Breeding Scheme implements androgenesis and protoplast fusion in addition to the techniques outlined by Chase. Initially, tetraploid clones are reduced to the dihaploid level through gynogenesis. Selected dihaploids are reduced to monohaploids via microspore culture, resulting in completely homozygous stable diploids when doubled. Following selection, diploids are sexually crossed and hybrids are selected. Selected diploid hybrids are then combined through protoplast fusion to create well-defined, heterotic, tetra-allelic cultivars which can be maintained through vegetative propagation or re-synthesized 
when necessary (Wenzel et al. 1979).

\subsection{Advantages of Haploid Breeding in Potato}

In a crop which is difficult to manipulate through conventional means, haploid breeding offers many advantages, a few of which are outlined below:

1. Homozygous breeding stocks of potato cannot be produced through inbreeding due to severe inbreeding depression in most tetraploids and self-incompatibility in most diploids. Therefore, the primary advantage of haploids is the efficient generation of homozygous clones through chromosome doubling of selected monoploids. Eventually, production of homozygous clones through haploid breeding may permit propagation of high yielding cultivars through true potato seed.

2. The tetraploid genome of potato is thought to harbor deleterious recessive alleles. When reduced to the monoploid level, there are no intra-allelic interactions, thereby exposing all genes to selective forces. Therefore, it can be inferred that genotypes with deleterious alleles will be eliminated.

3. The simplified genomic composition of doubled monoploids makes them useful for genetic analysis. For instance, there are fewer phenotypic classes owing to the absence of heterozygotes, the lack of dominance simplifies study of genetic effects, and doubled monoploids are highly homogeneous/stable resulting in increased experimental replicability.

4. Although gametoclonal variation occurs at a lower frequency than somaclonal variation, stable single gene mutations have been identified in androgenic populations. Thus the process 
of haploid induction through cell culture could potentially be used to create and introduce unique genetic variation into homozygous clones.

5. Transformation or mutagenesis of monoploids followed by chromosome doubling results in plants homozygous for the genetic alteration. Homozygosity is frequently prerequisite to expression of genetic mutations and gene insertions and is otherwise practically impossible to achieve without inbreeding which is not possible in many potato genotypes (Veilleux 1996, Wenzel et al. 1979, Foisset and Delourme 1996 and Evans et al. 1984 ).

\subsection{Prerequisites for Success}

In order for doubled haploid populations to be useful to plant breeders and geneticists, it is important that they be "comparable to conventionally derived lines" (Murigneux et al. 1993). For purposes of genetic studies, it is imperative that a random sample of gametes be regenerated. From a plant breeder's perspective it is desirable to generate homozygous lines which are representative of the full gametic array, in order "to fully exploit the genetic variability within a cross. Or at least, gametophytic selection should be in favor of agricultural fitness" (Tanhuanpaa 1994).

A doubled haploid population derived through microspore/anther culture or through gynogenesis is expected to "represent the segregation from the preceding meiosis." Although a randomly selected array of gametes is typically placed into systems of haploid induction, gametes may vary in their response to the system, resulting in the derivation of a population with skewed segregation for certain alleles. Selection pressures acting upon gametes in haploid induction systems may differ widely from selection pressures occurring during normal gametic 
development, pollination, and fertilization. Non-random allelic ratios (gametic selection) in doubled haploid systems could arise due to the following factors: preferential regeneration of gametes containing a higher percentage of alleles from the more androgenically/gynogenically responsive parent; linkage between genes for certain phenotypes and genes enhancing the androgenic or gynogenic response; gametic selection occurring in the gametophyte prior to cell culture; gametic selection against genotypes containing lethal or deleterious alleles; and gametoclonal variation enhancing the competitive ability of mutants (Logue 1996).

\subsection{Processes of Haploid Induction}

Haploid potato can be derived through two alternative processes: pollination of a maternal clone with a haploid inducing pollinator (gynogenesis) or microspore/anther culture (androgenesis). The gynogenic pathway of haploid induction is initiated by crossing the seed parent to the haploid-inducing pollinator resulting in a mixture of seeds containing hybrid and haploid embryos. Potential haploid seed are selected based upon a variety of morphological markers and germinated. Either flow cytometry or chromosome counts are employed to determine the ploidy level of putative haploids (Figure 1.2). The induction of androgenic potato haploids begins by picking buds when microspores are in the late uninucleate stage of development. After a 3-day cold treatment, buds are surface sterilized and the anthers are excised and cultured in flasks of MS (Murashige and Skoog 1962) liquid anther culture medium for 5-6 weeks in the dark on shakers. Embryos formed are harvested and plated on embryo regeneration medium. Regenerants are subcultured and embryos are transferred three times at 3- 
week intervals. The ploidy level is determined through flow cytometry or chromosome root tip counts (Figure 1.3).

\subsection{Gynogenesis}

\subsubsection{Haploid Frequency}

The first potato dihaploids obtained through gynogenesis resulted from a S. tuberosum $\times$ S. chaucha cross and were reported by Lamm in 1938. The following year a dihaploid was reported from a cross between S. tuberosum 'Aurora' and S. rybinii (Ivanovskaja 1939). After a period of little interest in potato haploids, a dihaploid of $S$. tuberosum 'Katahdin' was reported by a group of researchers at the University of Wisconsin in 1957 (Hougas et al. 1957). This was the first dihaploid reported in an effort to produce dihaploids on a large scale for evaluation of their potential in potato breeding. In 1958, the same group derived 28 dihaploids from 6,041 pollinations between 37 combinations of ten S. tuberosum and seven diploid Solamum species which produced 959 fully developed seeds and 806 viable seedlings, proving that interspecific (interploidy) crosses were a reliable means of producing potato haploids (Hougas et al. 1958). By 1960 , a population of 500 dihaploid $S$. tuberosum was developed. Monohaploids of $S$. tuberosum were first induced in 1975 from two cycles of gynogenesis. (A monoploid from a dihaploid had been previously reported by Frandsen in 1968, but its origin was uncertain). Crosses were made between a $S$. tuberosum dihaploid and a S. phureja haploid-inducing pollinator resulting in 6,383 seeds of which nine lacked the dominant embryo spot marker carried by the inducer. Of nine seeds, five germinated and three seedlings were monohaploid (van 
Bruekelen et al. 1975). Since then, numerous dihaploids and monohaploids have been induced from a wide variety of germplasm.

\subsubsection{Mechanism of the Pollinator Effect}

Early cytological observations of developing fertilized ovules from $4 x \times 2 x$ crosses showed that fertilization resulting in triploid embryos in pentaploid endosperm was generally lethal. Among 62 developing ovules, Wagenheim et al. (1960) found only one viable triploid embryo associated with pentaploid endosperm; 30 ovules contained hexaploid endosperm with no embryos; 26 tetraploid embryos with hexaploid endosperm; and five dihaploid embryos with hexaploid endosperm. The tetraploid embryos were proposed to arise from fertilization by unreduced pollen. He suggested two mechanisms of dihaploid origin: 1) a single sperm ( $2 n)$ from an unreduced haploid-inducer pollen grain may have fused with the secondary embryo sac nucleus and the other sperm was lost or absent; 2) fusion of two sperm (1n) with the secondary embryo sac nucleus by double fertilization. Both of these mechanisms would have resulted in the development of an unfertilized egg cell in viable hexaploid endosperm (Wagenheim 1960).

If fusion of a diploid $2 n$ sperm nucleus with the central cell of the megagametophyte results in parthenogenetic development of a dihaploid embryo with hexaploid endosperm, then superior pollinators should possess a high frequency of $2 n$ sperm nuclei. In order to test this hypothesis, Montelongo-Escobedo and Rowe (1969) studied two superior and two inferior haploid-inducers. Superior pollinators had 37.7 and $30.2 \%$ pollen tubes with single gametes (assumed to be restitution nuclei) whereas an inferior pollinator had $2.8 \%$ pollen tubes with single gametes. Colchicine treatment of pollen resulted in formation of pollen tubes with $100 \%$ restitution nuclei after 24 hours. When colchicine treated pollen was utilized in crosses, the 
number of haploids per 100 berries increased for both the superior pollinator and one inferior pollinator compared to the untreated pollen control. When treated, the other inferior pollinator produced 0.9 instead of zero haploids per 100 berries; however, this was not considered a significant increase. The observed positive correlation between $2 n$ sperm frequency and dihaploids induced per 100 berries provided indirect evidence for the role of restitution sperm nuclei in haploid induction (Montelongo-Escobedo and Rowe 1969). The proposed mechanism of monohaploid derivation differs only in that the secondary embryo sac nucleus is fertilized by a single reduced male gamete while the other gamete degenerates without fertilizing the egg cell (van Bruekelen et al. 1975).

Anueploids can result from the dihaploid induction procedure. Budin reported that $12 \%$ of the putative dihaploids they induced were actually anueploid (Budin 1969). The occurrence of anueploidy combined with recent evidence from molecular analysis suggests that a third mechanism may be involved in haploid induction. Dihaploid $S$. tuberosum plants induced by $S$. phureja were found to contain a percentage of aneusomatic cells with more than 24 chromosomes. From 17 clones, 15 were aneusomatic, five contained a small proportion of triploid cells, and two contained tetraploid cells. RFLP markers specific for the $S$. phureja inducer hybridized to the 15 aneusomatic clones but not to the two euploid clones. The authors suggested that the dihaploids may arise from triploid zygotes via preferential elimination of the inducer chromosomes in somatic cell divisions following fertilization (Clulow et al. 1991). Inducer-specific leaf isozymes and/or tuber patatin were found in five of eight dihaploids. The three dihaploids not expressing the genes had additional chromosomes (presumably from the inducer) in less than $15 \%$ of the observed cells. A positive correlation was seen between the 
percentage of aneuploid cells and the number and degree of inducer genes expressed, suggesting that the chromosomes in addition to the diploid number originated from the inducer parent (Clulow et al. 1993). A single dihaploid clone, which had been identified as euploid, was studied utilizing GISH (genomic in situ hybridization), isozymes, and RAPD markers. Of 24 RAPDs polymorphic between the seed parent and inducer, one band specific for the inducer was found in the dihaploid. An inducer specific isoform of alpha-esterase was also detected. GISH resulted in the detection of three large segments in the dihaploid which correlated with IvP48, the inducer parent (Wilkinson et al. 1995). Although this was initially taken as evidence for the introgression of inducer genes into seed parent chromosomes, the later discovery that the putatively euploid clone was actually aneusomatic shed doubt on the results/conclusions originally drawn from the GISH analysis (Allainguillaume et al. 1997). Principal component analysis of 21 morphological characteristics showed that the morphology of the dihaploids (all except one were known to be aneusomatic) was intermediate between the seed parent and the inducer. At least one inducerspecific PCR marker was detected in all the dihaploids, but the specific marker found varied among dihaploids. In addition all dihaploids exhibited at least one inducer morphological characteristic, which varied among dihaploids. The dihaploids formed a cluster between the seed parent and the inducer but were much closer to the seed parent, suggesting that the dihaploids expressed some inducer genes but were primarily influenced by the seed parent genome. From the evidence, it appears that segments of the inducer genome that were retained were randomly selected (Allainguillaume et al. 1997).

In conclusion, there is strong evidence for chromosomal elimination leading to aneusomatic dihaploids containing inducer chromosomes in addition to the seed parent 
chromosomal complement. However, many questions remain. It is unknown whether the majority of dihaploids is aneusomatic and whether monoploids are also aneusomatic. The alternative mechanisms of haploid formation have not been disproven and may occur in some cases. It remains to be determined whether recombination transpires between the parental genomes resulting in introgression of inducer genes into the seed parent chromosomes in addition to the occasional retention of entire inducer chromosomes and whether this event is random.

\subsubsection{Factors Affecting Frequency of Gynogenic Haploids}

\subsubsection{Genetic Control}

Early in the history of dihaploid induction through interspecific crosses it was suggested that the genotypes of both the tetraploid seed parent and the diploid haploid-inducer affected the rate of dihaploid induction (Peloquin and Hougas 1959). The significant effect of both the seed

and inducer parent upon dihaploid frequency was later systematically demonstrated by Hougas et al. (1964). In addition, a similar effect of both parental genotypes was evident in monoploid induction through gynogenesis (Uijtewaal et al. 1987).

Early investigators of gynogenic haploid induction were surprised to find that the pollinator affected the rate of haploid induction, since it was thought that the egg cell was not fertilized. Genotypic effects were then attributed primarily to the frequency of $2 n$ gametes or double endosperm fertilization. Pollination of a highly responsive seed parent, $S$. tuberosum 'Merrimac,' with ten different pollinators produced haploids in a range from 0.0 to 40.4 per 100 berries (Hougas et al. 1964). When pollinators with both high and low dihaploid inducing ability were crossed and/or selfed, both negative and positive transgressors were found in the resulting 
progeny, thus demonstrating genetic control and heritability of haploid inducing ability. This also indicates that haploid inducing pollinators could be bred with even greater haploid-inducing ability (van Bruekelen 1981).

In addition to the pollinator effect, it is evident that the seed parent exerts a great influence over the frequency of haploids and hybrids per 100 berries (Hougas et al. 1964, Liu and Douches 1993, van Bruekelen 1981, Caligari et al. 1988). For instance, variation was highly significant $\left(\chi_{(16)}^{2}=82.55, \mathrm{P}<0.001\right)$ for dihaploid frequency among the progeny of crosses between 17 different $S$. tuberosum clones and the haploid inducer IvP48. Seed parent influence could be exerted through a number of factors including genes for pseudogametic competency, cytoplasmic influences, deleterious alleles, or the number of ovules per berry and may be sporophytically or gametophytically determined (van Bruekelen 1981). van Bruekelen concluded that cytoplasmic influence was not important; however, his results may have been confounded by inbreeding depression. Based on inheritance studies, he suggested that the trait was primarily sporophytically determined with lethal alleles exerting a small influence on the gametophytic phase. The mode of inheritance appeared intermediate or dominant with many loci (van Bruekelen 1981).

\subsubsection{Environmental Factors}

Numerous authors have reported significant variation in haploid production frequency both within years (seasons) and across years. However, it is difficult to attribute the variation to a particular factor(s). Several possible factors including temperature, light intensity and duration, humidity, moisture availability, pesticide/chemical application, nutrient status, and physiological status and age have been implicated by various authors (Uijtewaal et al. 1987). In addition, the 
proficiency of personnel conducting pollinations and extracting, sorting, and germinating seed may also influence haploid frequency. Intra-seasonal variation was reported for one clone studied over an entire season, but the variation could not be correlated with temperature or light intensity in a period from 5 days before to 2 days after pollination. During a relatively warm season, haploid frequency was higher at the beginning of the season when temperatures were lower, though this effect may have been confounded by differences in plant age (Uijtewaal et al. 1987). Over a temperature range from $18-23^{\circ} \mathrm{C}$ there was no observed effect upon haploids per 100 berries $(\mathrm{dh} / 100 \mathrm{~b})$ in the maternal clone. However, higher seed set and more hybrids per 100 berries (hy $/ 100 \mathrm{~b})$ were observed at $18^{\circ} \mathrm{C}$ than at higher temperatures. Lower temperature $\left(18^{\circ} \mathrm{C}\right)$ had a positive effect on the pollinator in terms of $\mathrm{dh} / 100 \mathrm{~b}$ and hy/100b. The positive influence of lower temperature on $\mathrm{dh} / 100 \mathrm{~b}$ occurred within 2 days after transfer from high to low temperatures, unlike low temperature influence on hy/100b which occurred at least 15 days prior to pollination. It is likely that low temperature affects hy/100b during meiosis and affects $\mathrm{dh} / 100 \mathrm{~b}$ during final stages of pollen development, or during or immediately after pollination (van Breukelen 1981). Pollination of flowers differing in age of one clone on a single day demonstrated that the stigma is not receptive before petal coloration, but young open flowers are higher in dihaploid frequency as measured by $\mathrm{dh} / 100 \mathrm{~b}$ and also in hy/100b and percent fruit set than older flowers (van Bruekelen 1981). A pesticide, Dichlorephos, caused abortion of flowers pollinated 1 day prior to 4-5 days after pollination (van Bruekelen 1981) There is no research currently available on the effects of light intensity and duration, humidity, moisture and nutrient status, and chemical application upon haploid frequency. 


\subsubsection{Screening Techniques}

Products resulting from crosses between tetraploids and diploid haploid inducers are typically a mixture of tetraploids, rare triploids, dihaploids, and seeds lacking embryos. In monoploid induction from a diploid seed parent, products obtained are primarily diploid hybrids, a small percentage of monoploids, and seeds lacking embryos. In order to differentiate among the product classes without the time-consuming task of direct ploidy determination, several methods to screen for putative haploids have been developed based upon morphological markers. When testing interspecific hybridization as a means of deriving dihaploids, Hougas et al. (1958) utilized parents with strikingly different leaf morphology and/or pigmentation to facilitate selection of potential dihaploids (Hougas et al. 1958). In potato, two dominant genes designated as $P$ and $R$ result in purple and red pigmentation at nodes. When two other genes $B^{c}$ or $B^{d}$ are present in conjunction with $P$ or $R$ genes, a purple or red spot at the hypocotyl base of the embryo is visible through both sides of the seed coat. These genes are found in many $S$. phureja clones and infrequently in S. tuberosum selections. Presence of these genes in the haploid inducer allows elimination of the majority of hybrid progeny both at the seed and seedling stages. It was suggested that superior haploid-inducing pollinators be developed which were homozygous $P P B^{c} B^{c}, P P B^{d} B^{d}$, or $R R B^{d} B^{d}$ (Peloquin and Hougas 1959). Several authors were unsuccessful in developing such pollinators; however, Hermsen and Verdenius (1973) succeeded, enabling the induction of dihaploids and monoploids on a much larger scale. The superior pollinators were developed from $S$. phureja selections based on three criteria: high male fertility, homozygosity for the embryo spot marker, and ability to induce a large number of dihaploids per berry and per 100 seeds. The genes $P$ for 'colored hypocotyl' and $B^{d}$ for 'embryo spot' were selected as this 
particular combination resulted in a more pronounced embryo spot, hypocotyl coloration, and greater pleiotropic expression than the alternative allelic forms. Several pollinators of the genotype $B^{d} B^{d} P P$ with superior haploid induction capabilities (the IvP series) were generated and are now commonly used in haploid induction (Hermsen and Verdenius 1973). Budin (1969) suggested screening seeds from $4 x \times 2 x$ crosses for the embryo spot marker, germinating seeds lacking the embryo spot, and selecting seedlings without anthocyanin pigmentation at nodes. After passing through these screens, chloroplast counts per pair of guard cells were made, dividing plants into a group with more than 16 chloroplasts (non-haploid) and a group with less than 16 chloroplasts that consisted of a few tetraploids and triploids as well as the aneuploids and dihaploids. Chromosome counts were then made of the latter group which in his experiments constituted only $15 \%$ of those subjected to chloroplast counts, demonstrating the efficiency of this technique (Budin 1969). Within selected spotless seed, there is often a percentage of nonviable seed either lacking an embryo or possessing an underdeveloped embryo. Some seeds also have ambiguous spot markings which lead to misidentification. In order to reduce the percentage of non-haploid seed to be germinated, seeds can be sorted based on size in addition to spot marker. Caligari et al. (1988) experimented with 17 S. tuberosum clones crossed to the dihaploid inducer IvP48 and developed four classes: A) spot absent, diameter $>1.25 \mathrm{~mm}$; B) spot absent, diameter $<1.25 \mathrm{~mm}$; C) spot present, diameter $>1.25 \mathrm{~mm}$; and D) spot present, diameter $<1.25$ $\mathrm{mm}$. Mean days to germination (from earliest to latest) for the four groups differed and fell in the following order: $\mathrm{A}<\mathrm{C}<\mathrm{B}<\mathrm{D}$. The dihaploid seeds were the quickest to germinate, but there was significant overlap among the groups. Of the seeds in group A, $89 \%$ were dihaploid, whereas only $8.6 \%$ of the seeds in the remaining groups were dihaploid. In groups B and D 
(diameter $<1.25 \mathrm{~mm}$ ), $80.4 \%$ of the seedlings were triploid. Significant differences in ploidy level were found between spotted and spotless seed both across and within size groups. There were also smaller but highly significant differences in ploidy level based only upon seed size. Selection efficiency based upon spot absence was $85.6 \%$, based upon seed diameter was $68.6 \%$, and based on both criteria was $89 \%$. The percent of potential haploids obtained was $97.2 \%$ for spot absence, $96 \%$ for seed diameter, and $93.6 \%$ for both criteria. Although it was possible to reduce the number of seedlings to be evaluated cytologically, there was a slight increase in the number of haploids lost (Caligari et al. 1988). In dihaploid induction, a high percentage of haploids is obtained; therefore loss of a small fraction of the haploids may not be critical. However, the percentage of monoploids induced from diploid parents is much lower and any reduction in numbers obtained is highly undesirable. Liu and Douches (1993) crossed eight tetraploid clones with the $S$. phureja haploid inducer PI225682.22 (1.22), which is homozygous $P P$ for purple hypocotyl, a trait present in several of the seed parents. The inducer utilized did not possess genes for embryo spot $\left(B^{d}\right)$, therefore they screened the progeny for hybrids using morphological characteristics (anthocyanin pigmentation, high length to breadth ratio of the terminal leaflet, light green leaf color, reduced vigor, tuber color and isozymes). With two isozymes, $50 \%$ and $37 \%$ of the hybrids were identified, respectively. When combined with dosage effect data from the isozymes ( $61 \%$ identified), $80 \%$ of the total hybrids were identified. Phenotypic selection, excluding tuber color, resulted in $68 \%$ identification of hybrids, tuber color alone resulted in $46 \%$ identification of hybrids, and combining phenotypic selection with tuber color resulted in identification of $77 \%$ of the hybrids. When phenotypic analysis (including tuber color) was combined with isozyme analysis, $91 \%$ of the hybrids were eliminated. The PGM-2 
locus is present in the inducer but is rarely found in S. tuberosum selections; however, it is heterozygous in the inducer. Creation of an inducer homozygous for the $P G M-2$ locus as well as for genes controlling purple hypocotyl and embryo spot, would allow efficient identification of haploids at both seed and seedling stages. Several advantages of isozymes in haploid selection are their lack of ambiguity, availability of numerous different loci in potato, the small amount of tissue required which enables testing of small seedlings, and ease of use (Liu and Douches 1993). However, isozymes may lead to misidentification of dihaploids if small portions of the inducer genome (containing the isozymes) are incorporated into the dihaploid genome during chromosomal elimination.

In conclusion, gynogenesis is a simple technique of haploidization, though it may be labor intensive. The most likely mechanism of haploid formation appears to be normal fertilization followed by chromosomal elimination, though other possible mechanisms cannot be eliminated. Although frequency of haploid induction is influenced by environmental factors, conducive conditions have been difficult to define. In general, conditions favorable for plant development are also favorable for haploid induction. Selection of seeds based on embryo spot and other pigmentation markers is the most effective means of identifying the haploids. Gynogenic haploid frequency is heavily dependent upon the seed and pollen parent genotypes. Although it appears that there are specific genes for gynogenic haploid development, the flower set and seed production capabilities of the maternal clone can be a limiting factor. It appears that there are genotypes which do not produce haploids through this mechanism; however, additional efforts may prove elsewise. It is possible to develop both seed parents and pollinators that produce high frequencies of haploids when crossed with a wide range of other genotypes. 
Breeding for haploid inducing ability in pollinators has been successful, and it is possible that further progress could be made.

\subsection{Androgenic Pathway}

\subsubsection{Haploid Frequency}

Haploid induction via anther culture, first reported in Datura innoxia, was generally extremely successful with other members of the Solanaceae. However, success with potato was not as readily achieved (Dunwell and Sunderland 1973). Haploids of tuber-bearing Solamum were first reported in 1971 and 1972. Irikura and Sakaguchi (1972) reported haploid induction from $S$. verrucosum $(2 n=2 x=24)$, though anther culture of dihaploid $S$. tuberosum was unsuccessful. Dunwell and Sunderland (1973) reported callus and embryoid formation from anthers of $4 x S$. tuberosum; however, they did not regenerate haploids. Sopory and Rogan (1976) cultured approximately 25,000 anthers from 34 dihaploid clones of S. tuberosum on 92 different media. Callus was induced from 20 clones and embryos from five. In 1977, Sopory reported that a total of 31 plants had regenerated from two clones, though none were monoploid. Foroughi-Wehr et al. (1977) regenerated monoploid plants from one of six cultured dihaploid $S$. tuberosum clones. In recent years, anther culture success has improved for commercial tetraploid S. tuberosum clones. Rokka et al. (1996) reported of 40 tetraploid potato clones, 28 formed embryos and 17 formed shoots. Embryos per 100 anthers ranged from 0.1 to 45 and shoots per 100 anthers ranged from 0.1 to 5.3 . 


\subsubsection{Mechanism of Haploid Induction}

The developmental pathway from microspore to haploid plantlet remains even more of an enigma than the gynogenic pathway. There are three alternative primary initiation pathways of androgenesis according to Meyer et al. (1992):

I. The first mitotic division of the nucleus proceeds normally followed by degeneration of the generative nucleus. This induces mitotic division of the vegetative nucleus leading to appearance of multinuclear stages.

II. The first mitotic division of the microspore nucleus is replaced by symmetric divisions which form a multicellular microspore with one nucleus per cell.

III. Symmetric nuclear divisions occur instead of the normal first mitotic division. However, multinuclear microspores are generated rather than multicellular microspores, as seen in the second pathway.

The second pathway is the most desirable, since it leads to the formation of haploid structures (Meyer et al. 1992). Unfortunately, the mechanism determining which androgenic initiation pathway occurs remains unknown for potato.

In addition to the existence of multiple initiation pathways of androgenesis, there are two different routes of further growth leading to plantlet formation in potato androgenesis. Continued microspore development leads to the formation of embryos which mature into plantlets through developmental stages similar to zygotic embryos, or callus proliferates from microspores within the anther and later regenerates through growth regulator manipulations (Reynolds 1990). It is also possible to obtain both embryos and callus from the same anther, since the response is influenced by culture conditions, especially plant growth regulators, as well as by genotype. 
Early observations of embryogenesis revealed that equal cell division occurs 2-4 days after culture initiation leading to development of a multicellular structure. The multicellular structures are released from the exine after 8-10 days; and within 19-22 days, embryos break through the anther wall (Sopory et al. 1978). In the alternative developmental route, callus (which can proliferate both from microspores and from somatic anther tissues) typically appears within several weeks of culture and may regenerate after several passages on a shoot induction medium. Embryogenesis is generally considered superior to callus induction as a means of inducing haploids from potato anther cultures for several reasons. The callus phase may give rise to greater somaclonal variation, especially with regard to change of ploidy. In anther-derived callus obtained from five dihaploid clones, aneuploidy as well as polyploidization with chromosome numbers ranging from 12 to over 200 have been noted. Abnormalities such as polytene chromosomes, dicentric chromosomes, and lagging chromosomes were also observed (Sopory and Tan 1979). In addition, the point of origin of callus frequently cannot be deduced, but proliferation from somatic tissue of the anther would give rise to somaclones (Tiainen 1992b).

\subsubsection{Factors Affecting Haploid Frequency}

\subsubsection{Genotype}

Since the advent of potato anther culture, strong genotypic effects on anther culture response have been noted both in callus production and direct embryogenesis. This has resulted in highly variable responses among genotypes after application of seemingly identical anther culture procedures and has made the definition of a single efficient anther culture protocol difficult. In addition, complete lack of response from certain genotypes narrows the range of 
genetic material from which haploids can be extracted, reducing the value of this technique in plant breeding. In general, extraction of monoploids from diploid and dihaploid clones has been easier than extraction of dihaploids from tetraploid S. tuberosum (Tiainen 1992b).

Study of callus induction from whole stamens in several potato species and hybrids demonstrated that callus growth type (none, filament, or anther) in hybrids was determined by the paternal parent, suggesting control by a male cytoplasmic factor or expression of paternal genes for callus induction. The callus originating from anthers was thought to be gametophytic in origin, but due to lack of morphogenesis, this could not be verified (Simon and Peloquin, 1977).

Genetic control over androgenesis has been clearly demonstrated; however, there are conflicting reports on the number of loci controlling the response and whether alleles for embryogenesis are dominant, co-dominant, or recessive. When poorly to moderately responsive genotypes were selected and crossed, progeny were obtained with much greater embryogenic capacity than the parents, demonstrating inheritance and recombination of genes controlling embryogenesis in potato anther culture (Jacobsen and Sopory 1978). Crossing diploid plants with no embryogenic response with a clone with high response yielded progeny with a wide continuous range of responses. Wide variation in response was also seen when the $F_{1}$ were backcrossed to the unresponsive parent. This indicated that embryogenesis is controlled by genes at more than one loci. Recessive or complementary gene action appeared likely as crossing two non-responsive genotypes yielded responsive genotypes (Sonnino et al. 1989). Uhrig (1985) conducted anther culture on 10 randomly selected diploid S. tuberosum clones. From the highly responsive clones (determined by plantlets/100 anthers) diploid regenerants were selected for anther culture. Selection was conducted for two cycles and the mean response of the unselected, 
first cycle, and second cycle were compared. Embryo production significantly increased between the unselected and first cycle clones, and percent regeneration increased in between both cycles. Moderate anther culture competence was displayed in the $\mathrm{F}_{1}$ resulting from a cross between a responsive and non-responsive clone of $S$. phureja. The segregation ratio of absence:presence of androgenic competence in the $F_{2}$ was $12: 3$ which is not significantly different from the $3: 1$ segregation ratio expected in traits controlled by single genes expressing complete dominance. Other genes with reduced effect may modify the level of response (Taylor and Veilleux 1992). Anther culture competence inheritance studies of hybrid families derived from anther culture competent S. phureja and incompetent S. phureja, S. microdontum, and S. berthaultii indicated that androgenesis may be under the control of a single gene with dominant action (Singsit and Veilleux 1988). From three cycles of selection for production of plantlets through embryogenesis, Uhrig (1985) obtained ten $2 x$ clones that were designated EAPP (efficient anther plant producing clones) and doubled to obtain $4 x$ EAPP clones. Crossing of $4 x$ EAPP clones with non-responsive tetraploid genotypes gave rise to plants with low, moderate or higher response compared with the EAPP parent. This again suggested simple inheritance by gene(s) with dominant action (Uhrig and Salamini, 1987).

A small proportion of the regenerants selected from highly responsive donors had reduced embryogenic capacities (Uhrig 1985). Generation of genotypes through anther culture with both higher and lower rates of embryogenesis than the donor clone was also reported by Veilleux et al. (1985), and is indicative of partial sporophytic control over anther culture response (Uhrig 1985). Independent responses of embryogenesis and embryo regeneration to selection suggested that capacity for embryo production and regeneration may be independently controlled (Uhrig 
1985). Lack of correlation among the same populations for anther culture, leaf disc regeneration, and protoplast culture competence indicated that responses to different tissue culture procedures may be inherited independently (Taylor and Veilleux 1992). The ability to select and breed for embryo regeneration capacity was demonstrated by Cappadocia et al.(1984). In contrast to embryogenesis, transfer of regenerative capacity was only effective in specific genotypic combinations.

Positive transgressants of the better parent for anther culture response have appeared in the $F_{11}$ progeny of crosses between responsive and non-responsive parents (Uhrig and Salamini 1987; Sonnino et al. 1989). This indicates heterosis for anther culture response, or that the nonresponsive parent contributed positive alleles for anther culture response.

Although the details of genotypic influences upon anther culture response are ambiguous, several key points are apparent. First, callus formation, embryogenesis, and regeneration are genetically determined and may be controlled by independent loci. Second, there are both sporopytic and gametophytic determinants. Third, heterosis appears to increase embryogenesis. Fourth, and of tremendous importance to plant breeders, crossing plants with high androgenetic capacity to desirable but unresponsive genotypes should generate plants which combine high androgenetic capacity with the desired trait. The success of this strategy has been demonstrated by Uhrig (1983) who combined androgenic response with Globodera pallida resistance.

\subsubsection{Environmental Influence on the Donor Plant}

In addition to genotype and factors acting directly upon anthers/microspores during culture, the physiological condition of the donor plant and environmental conditions during preculture phases of microspore development can influence embryo and callus development and 
subsequent shoot regeneration. High levels of $\mathrm{CO}_{2}$ around the donor plant decreased flowering and androgenic response of S. tuberosum (Johansson 1988). Approximately twice as many dividing microspores were seen in field-grown S. chacoense 'IP33' compared to anthers from plants grown in a greenhouse (Cappadocia et al. 1984). In contrast, growth of S. tuberosum anther donors in the field or greenhouse did not have a significant effect upon androgenic response (Cappadocia and Ahmin 1988). Tremendous seasonal variation has been observed in anther culture experiments. For instance, responsive anthers ranged from 15-20\% in September and October to 1-3\% between February and May (Tiainen 1992b). Seasonal fluctuation was also observed in $S$. phureja 'PP5,' with the best anther culture response correlating with periods of the most vigorous plant growth and flowering (Veilleux et al. 1985). When $S$. phureja donor plants were grown under 14 or $18 \mathrm{~h}$ photoperiods for $8,10,12$, or 14 weeks, maximal embryo and plantlet formation was observed with young plants ( 8 weeks) and moderate photoperiod (14 h)

(Owen et al. 1988). Generally, maximal anther culture response is achieved on plants grown under favorable environmental conditions.

\subsubsection{Microspore Stage}

Early in the development of potato anther culture technique, it was noted that the stage of microspore development affected frequency of embryo and plantlet induction. Embryo formation was noted predominantly from anthers in the meiotic stage, some from the microspore stage, and a very few from the young pollen grain stage. It was suggested that the stage-requirement is more critical than the medium composition (Dunwell and Sunderland 1973). The uninucleate pollen stage was identified as the most responsive to anther culture in several systematic studies 
(Sopory and Rogan 1976, Sopory et al. 1978), though other authors have noted no difference between the uninucleate and tetrad stages.

\subsubsection{Medium}

\subsection{Salts and Gelling Agents}

Early anther culture experiments were conducted primarily on solidified medium using a wide variety of growth regulators and basal media. In general, variations of Murashige and Skoog (MS) (1962), Nitsch and Nitsch (1969), and Linsmaier-Skoog (1965) media were found to be effective though, as Irikura stated in 1975, "the frequency of plantlet formation varied together with the species, the clones, and the media." The polyploid species of the Demissa and Longipedicellata series responded best to Nitsch and Nitsch medium, whereas species from all the other series favored modified MS medium (Irikura 1975). S. tuberosum monoploids were derived on Linsmaier-Skoog medium from callus growth (Foroughi-Wehr et al. 1977) and have been obtained through direct regeneration of embryos on modified MS medium as well (Sopory et al. 1978). The Mexican potato species, S. fendleri and $S$. stoloniferum, both produced haploids on both modified Wenzel and modified Nitsch medium (Singsit and Hanneman 1987). Recently, anther culture systems employing bi-layer or liquid medium have been utilized in addition to solid medium. Culturing S. tuberosum anthers on Linsmaier-Skoog liquid medium was found to increase embryogenesis over culture on solid medium for many $2 x$ interspecific hybrids (Uhrig 1985). A bi-layer system with anthers floating in a liquid layer of modified MS medium over agar-solidified modified MS medium with activated charcoal was developed by Johansson (1983) and was found to induce embryos in 19 of 20 tested $4 x$ S. tuberosum clones (Johansson 1986). In a recent study of modified MS solid medium (Wenzel and Uhrig 1981), the double- 
layer system of Johansson (1986), Uhrig's (1985) liquid Linsmaier-Skoog modification, and $\mathrm{A}_{5} \mathrm{CA}$ liquid medium (Cappadocia and Ahmim 1988) with five tetraploid commercial $S$. tuberosum cultivars, four clones responded best to solid MS medium (Wenzel and Uhrig 1981) and one gave the best response on Uhrig's (1985) medium (Tiainen 1992b). Frequently, as evident above, interactions between culture systems and genotypes are too complex for broad generalizations.

It has been suggested that microspore culture has several advantages over culture of whole anthers, especially by elimination of embryogenesis from somatic tissue. Embryos have formed in isolated microspore culture of $S$. tuberosum cultivars, indicating that it may be possible to generate dihaploids through this mechanism. However, further refinement of this system is needed as plantlets have not been regenerated (Bugarova and Pretova, 1996).

In a bi-layer anther culture system, potato starch was found to be superior to gellan gum in embryo initiation during the first part of the culture period; however, gellan gum surpassed potato starch by the end of the culture phase. It was suggested that enzymatic breakdown of the potato starch liquified both layers of the medium, causing oxygen deprivation and reduced light availability (Calleberg et al. 1989). Addition of $0.35 \%$ Gelrite to medium with $3 \%$ potato starch resulted in the highest embryo production throughout the entire culture period. The Gelrite maintained solidification of the medium throughout the culture phase, whereas potato starch is thought to exhibit some nutritive value (Calleberg and Johansson 1993)

\subsection{Growth Regulators}

There is much contradiction between early and later papers regarding use of growth regulators in anther culture. In the early papers, authors frequently tried to induce callus growth, 
which differs from gametic embryogenesis in growth regulator requirements. In this section, I will only address growth regulators with regard to embryogenesis. In one experiment, BA ( $\mathrm{N}^{6}$ benzyladenine) appeared to be the most effective and the ideal concentration to be $4 \times 10^{-6} \mathrm{M}$ in combination with IAA (indole-3-acetic acid) at a concentration of $6 \times 10^{-6} \mathrm{M}$. BA was not tested alone in this experiment, but it was suggested that IAA was not as important (Sopory et al. 1978). For dihaploid S. tuberosum clone H3-703, IAA and BA at concentrations of $6 \times 10^{-6} \mathrm{M}$ and $4 \times$ $10^{-6} \mathrm{M}$, respectively, were superior to IAA or BA independently and to IAA in conjunction with zeatin or kinetin or BA in conjunction with NAA ( $\alpha$-napthaleneacetic cid) or 2,4-D (2,4dichlorophenoxyacetic acid). Addition of 2,4-D resulted in undesirable callus growth (Sopory 1979). In experiments with the tetraploid clone 'Pito' the growth regulators 2,4-D, zeatin, GA (gibberellic acid), and ABA (abscisic acid) either had null or negative effects on embryogenesis. IAA was beneficial at $0.02 \mathrm{mM}$ when used alone but had a negative effect when combined with BA. BA at $0.013 \mathrm{mM}$ during anther culture promoted the highest rates of embryogenesis and plantlet regeneration (Tiainen 1993). In general, IAA or BA together or independently appear beneficial but not absolutely required in anther culture of potato.

\subsection{Carbon Source}

Sucrose concentration was optimized at $60 \mathrm{~g} / \mathrm{L}$ for solid defined culture medium (Sopory et al. 1978). When P59 (defined MS-based medium) was used, optimal sucrose concentration was $6 \%$. In contrast, P58 medium (potato extract + growth regulators $+0.05 \%$ activated charcoal) reached maximal embryo production with $2 \%$ sucrose. In addition, the high sucrose $(6 \%)$ is only necessary for the first 6-8 days for maximal embryogenesis after which $2 \%$ sucrose is as effective. Glucose could not replace sucrose, but addition of glucose with sucrose 
augmented embryogenesis (Sopory 1979). When sucrose was replaced with an equimolar concentration of maltose, embryogenesis was reduced in a highly-responsive clone; but embryo regeneration was greatly increased. However, these results may not be generally applicable across genotypes; and the authors suggested that reduced embryo competition for nutrients may have been the primary factor causing the response and not intrinsic chemical differences between maltose and sucrose (Batty and Dunwell 1989). In contrast, pure sucrose at $60 \mathrm{~g} / \mathrm{L}$ gave the highest embryo and plantlet production rates in S. tuberosum 'Pito,'when compared with maltose, melibiose, and mannitol at three different concentrations alone or in conjunction with sucrose at $60 \mathrm{~g} / \mathrm{L}$. Melibiose and mannitol were significantly inferior to sucrose in plantlet production. Maltose at $60 \mathrm{~g} / \mathrm{L}$ was second to sucrose in plantlet production, but was significantly inferior (Tiainen 1992). It appears that genotype determines whether sucrose or maltose is superior for plantlet production.

\subsection{Other Components}

Addition of potato extract to the medium was found to be beneficial for embryogenesis but not necessary (Sopory et al. 1978). In $S$. carolinense, calcium $\left(\mathrm{CaCl}_{2}\right.$ at $\left.3 \mathrm{mM}\right)$ greatly increased both embryogenesis in combination with IAA and callus formation in combination with 2,4-D. When combined with IAA, $\mathrm{Ca}^{2+}$ induces ethylene synthesis which was suggested to increase embryogenesis. However, calcium clearly increases androgenic callus production with 2, 4-D, though ethylene production is not stimulated, suggesting that calcium is involved in signal transduction leading to androgenesis (Reynolds 1990). In addition, ethylene has been indirectly demonstrated to reduce embryogenesis. Addition of ethrel, which releases ethylene, greatly reduced embryogenesis, whereas a precursor in the biosynthetic pathway, methionine, had 
no effect. Ethylene inhibitors, polyamines, and reducing agents, which should decrease ethylene production, in general greatly increased embryogenesis; though a few had little effect (Tiainen 1992a). Activated charcoal at $5 \mathrm{~g} / \mathrm{L}$ was necessary for high anther culture response (Sopory et al. 1978, Sopory 1979). Presumably, activated charcoal acts by removing inhibitory substances from the anthers or culture medium (Sopory 1979).

\subsubsection{Pretreatment and Environmental Conditions}

Pretreatment of anthers for 2 days at $6^{\circ} \mathrm{C}$ increased embryogenesis or had no significant effect depending on the genotype, whereas pretreatment at $30^{\circ} \mathrm{C}$ had a negative effect (Tiainen 1992b). In a study of four $4 x$ genotypes, $25^{\circ} \mathrm{C}$ incubation temperature increased embryogenesis in three clones compared with $20^{\circ} \mathrm{C}$. A positive correlation was seen between optimal pollen germination temperature and optimal culture temperature for the three clones responding to high culture temperature (Calleberg et al. 1989). Later, the same authors reported that although the most effective temperature for embryogenesis and regeneration varied with genotype, $20^{\circ} \mathrm{C}$ generally gave the highest embryo regeneration rate (Calleberg and Johansson 1993). Light was found to increase embryogenesis at 2,000 lux for $12 \mathrm{~h}$ during anther culture on solid medium (Sopory et al. 1978).

In conclusion, androgenesis is a genetically controlled response to an in vitro process. In addition to genotypic effects, condition of the anther donor, anther pre-treatment, microspore stage, medium components, and environmental conditions during culture can influence the production of callus or embryos and their subsequent regeneration.

\subsection{Equivalency of Androgenic and Gynogenic Haploid Populations}




\subsubsection{Evidence from Other Crops}

As seen above, there are obvious differences between the processes of derivation of androgenic and gynogenic haploids. First, gynogenic monoploids are derived from egg cells through in vivo processes resembling normal gametic development, with the exception of exclusion or elimination of the paternal genomic contribution. In contrast, androgenic monoploids are derived exclusively through in vitro culture of uninucleate microspores whose developmental pathway is drastically altered from normal release and transfer, certation, pollen tube development, and fertilization to develop through embryogenesis into a sporophyte. There are similarities between the mechanisms as well as contrasts. In both systems, only a small percentage of the gametes exposed to the induction treatment develop into haploids. Both processes are genetically controlled and are greatly influenced by environmental conditions as well. The end results of androgenesis and gynogenesis are the same: haploid plants, or sporophytic expressions of single gametic genomes.

Given that meiotic processes generate male and female gametes with equivalent total genotypic arrays, it is expected that androgenic and gynogenic populations derived from the same clone would also be indistinguishable. However, evidence from other genera suggests that there may be intrinsic differences between male and female gamete-derived populations. Several studies of androgenic and gynogenic doubled haploid populations have indicated that gynogenically-derived lines are agronomically superior. A study of androgenic (ADH) and gynogenic (GDH) doubled haploid tobacco lines demonstrated that ADH were significantly less vigorous than GDH and a conventionally-derived inbred population (Kumashiro and Oinuma 1985). Comparison of barley lines generated through single seed descent (SSD), microspore 
culture (ADH) and the H. bulbosum (MDH) technique found that the mean values of agronomic traits were similar in the SSD and MDH populations, but were significantly lower in the ADH population (Powell et al. 1986). In contrast, several studies have demonstrated that androgenic and gynogenic monoploids derived from the same parent are not significantly different, or that there is no general trend in relative vigor. Burley tobacco MDH and $\mathrm{ADH}$ populations had essentially equivalent performance, though both expressed variation not seen in the progeny of selfed inbred lines (Nielsen and Collins 1989). Examination of seed set and winter hardiness in anther-derived and H. bulbosum derived doubled haploid lines of winter barley revealed no significant differences (Devaux 1988). It was concluded from a comparison of barley MDH and ADH populations that the effects of method differed among genotypes and crosses even within a species. Although the genotypic array derived through various mechanisms from the same barley genotype may differ, no mechanism of haploid induction was generally superior for all crosses (Bjornstad et al. 1993). These studies demonstrate the presence of discrepancies regarding relative value of maternally and paternally derived doubled haploids among and occasionally within crop species. In general, androgenic populations are equal or inferior to gynogenic doubled haploid populations; however, androgenesis is frequently a more efficient derivational process. Therefore, determination of the best mechanism of haploidization must be conducted independently for each crop species.

\subsubsection{Recombination}

Differences in recombination rates between male and female gametes have been noted in numerous animal and plant species. In humans and many animal species, excess recombination over the entire genome is often observed in females, whereas excessive recombination in males is 
limited and is often observed in specific genomic regions such as the telomeres. A map of the entire swine genome showed greater recombination in sowss than boars $(p<0.001)$ and a mean ratio of female:male recombination at 1.4:1 (Marklund et al. 1996). A consensus linkage map for chromosome 7 of swine was $153.3 \mathrm{cM}$ in males but $215.3 \mathrm{cM}$ in females (Roher et al. 1997). Regions of swine chromosome 1 arm q were identified with excess recombination in males, though there was excess recombination in females in the terminal regions of both arms. For $80 \%$ of swine chromosome 1, $1 \mathrm{cM}$ was estimated to equal 2-4 Mb; but in the terminal region of arm q, $1 \mathrm{cM}$ equaled $0.4 \mathrm{Mb}$. (Ellegren et al. 1994). Linkage maps of bull and cow had similar total lengths; however, individual chromosome lengths varied considerably between the two maps (Barendse et al. 1997). Female baboons have significantly higher recombination rates $(\mathrm{P}=0.017)$ than males with a genome map length estimated to be three times longer (Rogers et al. 1995). Mice also exhibit excessive female recombination (Blank et al. 1988). The total length of the human genome map for females is $4,396.9 \mathrm{cM}$ in contrast to the male map of 2,729.7 cM (Dib et al. 1996.)

In plants, excess recombination is often observed in both male and female meiosis, in contrast to most animals. Devaux et al. (1995) compared linkage maps derived from antherderived doubled haploids (ADH) and maternally-derived doubled haploids (MDH) of a 'Steptoe' $\times$ 'Morex' barley hybrid. An 18\% average increase in recombination in the ADH map as opposed to the MDH map was found. Most of the increased male recombination occurred in the telomeric regions (Devaux et al. 1995). Maps of an $F_{1}$ between a male and female clone of cassava (Manihot esculenta Crantz) also showed more recombination in the male parent (Fregene et al. 1997). In Brassica nigra, enhanced male recombination was found in the sub-telomeric 
and telomeric regions of chromosomes and excessive female recombination was located proximal to the centromeric regions (Lagercrantz and Lydiate 1995). Maps of four wheat chromosomes based on male and female derived doubled haploid populations revealed significantly greater male recombination on chromosome 7D and significantly enhanced female recombination on chromosome $7 \mathrm{~A}$. In general, higher recombinational frequencies were observed for the same sex within a chromosome, but differed among chromosomes (Wang et al. 1995). A study of a hybrid and backcross population of $S$. tuberosum $\times$ S. spegazzinii revealed reduced recombination in the male parent; however, this could have been influenced by the interspecific nature of the populations (Kreike and Stiekema 1997). Linkage map development of androgenic, selfed, and hybrid progeny of two Solanum chacoense clones revealed less recombination in the male gametes ( $206 \mathrm{cM}$ total map length) than in the female gametes (375 $\mathrm{cM}$ total map length) of one clone throughout the entire genome. Maps based upon male and female progeny derived from the other clone were approximately equal in length though some regions were longer in both populations.

Recombinational differences have also been observed due to position, age, and temperature. Excessive recombination in telomeric regions has been observed in species such as Drosophila, humans, and swine. The extent of excessive telomeric recombination is often also sex-specific (Ellegren et al.1994). Analysis of human chromosome I as a function of parental age revealed that recombination significantly decreased with increasing age in the female parent. In addition there was a decrease in numbers of double crossovers between two younger age groups and the older age group (Tanzi et al. 1992). 
What causes differential recombination rates? This question remains largely unanswered, though development of high density linkage maps may provide some insight in the near future. The presence of differences in recombination rates based on sex appears ubiquitous throughout the major taxa. However, the direction of excess recombination varies among species and within particular genomic regions. The ability to define recombinational characteristics within a species, which are different from the recombinational characteristics of other species, indicates that recombinational frequency is not a random element but a controlled event. Perhaps the genome itself contains elements which regulate recombinational frequency in conjunction with environmental stimuli. To a plant breeder, recombinational differences achieve meaning when attempting to break linkage between desirable and inferior traits. Although recombination does not normally create new allelic forms, higher recombinational rates translate into greater diversity in the entire spectrum of allelic combinations within a population.

\subsubsection{Selection}

Gametic selection is broadly defined as the action of selective forces on gametic tissue. In naturally-occurring systems, it is the result of action upon the male or female gametic tissue between the formation of the gametes and their union to form the zygote by any force which favors one allelic form over the alternative allele. In normal reproductive processes, gametic selection is most often attributed to selective pressures exerted upon the male gametophyte by the gynoecium between pollination and fertilization including self incompatibility reactions, environmental influences, and differential competitive ability of pollen grains (Xu et al. 1997). In haploid derivation, gametic selection is re-defined as the ability of an individual male or female gamete to respond to haploid-inducing stimuli, alter their developmental course, and 
succeed as sporophytes. This ability is contingent upon presence of alleles for androgenesis and gynogenesis and absence of deleterious and lethal alleles. Gametic selection is primarily observed as deviations from the expected Mendelian allelic ratios in linkage maps. There are numerous examples of distorted segregation in anther-derived haploid populations, i.e., maize (Murigneux et al. 1993), barley (Zivy et al. 1992), rice (Xu et al. 1997), and rape seed (Tanhuanpaa et al. 1994). However, few papers on this topic compare distortion in androgenic and gynogenic haploid populations derived from the same parental genotype. In linkage maps of barley populations which were derived through anther culture and the $H$. bulbosum method (gynogenesis), the total number of skewed loci was only slightly higher in anther-derived population; however, the extent of the deviation was much greater than in the gynogenic population (Devaux et al. 1995). A segment of one chromosome in a map of four wheat chromosomes showed significant distortion in the androgenic population, but not in the gynogenic population (Wang et al. 1995). These papers suggest that different loci may have skewed segregation ratios in anther-derived and gynogenic populations, and that the extent of segregation distortion may also differ between the populations. Since both androgenesis and gynogenesis are genetically controlled, it is expected that loci influencing these traits will show segregation distortion in the resulting haploid populations and that the particular distorted loci may differ between androgenic and gynogenic populations. Loci at which the parental clone is heterozygous for lethal or deleterious alleles would also be distorted, but this distortion would occur in the same direction in both (Rivard et al. 1996). 


\subsubsection{Gametoclonal Variation}

Gynogenic monoploids can be generated entirely in an in vivo system; in contrast, derivation of androgenic monoploids requires a minimum of 2 months in tissue culture. This provides opportunity for gametoclonal variation to arise in anther-derived haploids.

Gametoclonal and somaclonal variation can give rise to useful genomic changes; however, the majority of documented alterations have had negative effects. (For an excellent review of somaclonal variation, see Veilleux and Johnson 1998). Anther-culture induced heritable changes in the nuclear genome of two different tobacco species were independently demonstrated. Obvious phenotypic differences were observed between the highly inbred tobacco cv. Coker 139 and doubled haploids derived from it. Many anther-derived progeny were significantly inferior to the parent for yield and several other agronomic characteristics. Analysis of the nuclear genome of the parent, the doubled haploids, and their selfed progeny showed a significant increase in total nuclear DNA content of all the doubled haploid lines and progeny. It appeared that the increase in DNA occurred in the heterochromatic regions (Dhillon et al. 1983). A separate investigation of Nicotiana sylvestris doubled haploids and selfed progeny of the doubled haploids showed similar results. The doubled haploids were inferior to the parent and many exhibited an abnormal leaf type. An increase was seen in the total amount of nuclear DNA and in the proportion of repetitive DNA content with increasing anther culture cycles. Heritability of these changes was demonstrated, though reversions were seen in some progeny (De Paepe et al. 1982). An anther-derived monoploid of Solanum phureja had an increase in rRNA gene copy number compared with the diploid parent and altered restriction enzyme recognition sites. In addition there was a higher proportion of highly repetitive DNA than in the anther donor, 
suggesting occurrence of DNA amplification (Pehu 1986). It was suggested that differential DNA amplification in the microspore's vegetative nucleus, which does not occur in the generative nucleus, may cause the apparent anomalies between DNA content of anther donors and doubled haploid progeny (De Paepe et al. 1982; Dhillon et al. 1983). Gametoclonal variation is typically not phenotypically detectable in anther-derived potato plants, and is unlikely to be a significant concern in the use of anther-derived lines in potato breeding. In cases where frequent undesirable variation is seen, other mechanisms of haploidization or other breeding schemes should be investigated. Potato haploids derived through gynogenesis are not expected to exhibit gametoclonal variation, since they arise through an embryonic and seed developmental pathway.

\subsection{Concluding Statement}

Currently, both androgenic and gynogenic dihaploid and monoploid potato are being utilized in breeding programs. Although both methods result in production of haploid sporophytes, presumably arising from the same gametic array regardless of the mechanism used, the two mechanisms differ tremendously. Differences may arise between populations derived through the alternative mechanisms due to presence of haploid-inducer genes in gynogenic plants, dissimilar recombination rates in male and female gametes, differences in selection pressures, and somaclonal variation during androgenesis. In order to determine if one method of haploidization gives rise to superior or different genotypic arrays, it is necessary to compare the morphological traits and/or agronomic characteristics of the resulting populations. Such a comparison of haploid potato populations resulting from the two mechanisms, which is of significance to any potato breeding program utilizing haploids, has not yet been conducted. 


\subsection{Research Objectives}

Our primary objectives were to derive populations of monoploids from clones of Solanum phureja through the two alternative mechanisms of androgenesis and gynogenesis. We also wished to compare their morphological characteristics and agronomic traits in a greenhouse study to determine if one mechanism produced significantly better or different arrays of monoploid genotypes. In addition, derivation of these experimental populations will facilitate comparison of recombination rates and allelic segregation ratios between male and female gamete-derived populations, determination of the presence and frequency of genes originating from the haploidinducing pollinator in the gynogenic monoploid genomes, and may lead to further elucidation of the gynogenic mechanism in potato. 


\section{BIBLIOGRAPHY - CHAPTER I}

Allainguillaume, J., M. J. Wilkinson, S. A. Clulow and S. N. R. Barr, 1997: Evidence that genes from the male parent may influence the morphology of potato dihaploids. Theor. Appl. Genet. 94, 241-248.

Barendse, W., D. Vaiman, S. Kemp, Y. Sugimoto, S. Armitage, J. Williams, H. Sun, A. Eggen, M. Agaba, S. Aleyasin, M. Band, M. Bishop, K. Byrne, F. Collins, L. Cooper, W. Coppettiers, B. Denys, R. Drinkwater, K. Easterday, C. Elduque, S. Ennis, G. Erhardt, L. Ferretti, N. Flavin, Q. Gao, M. Georges and A. Van-de-Weghe, 1997: A medium-density genetic linkage map of the bovine genome. Mam. Genome 8, 21-28.

Batty, N. and J. Dunwell, 1989: Effect of maltose on the response of potato anthers in culture. Plant Cell Tiss. Org. Cult. 18, 221-226.

Bjornstad, A., H. Skinnes and K. Thoresen, 1993: Comparisons between doubled haploid lines produced by anther culture, the Hordeum bulbosum-method and lines produced by single seed descent in barley crosses. Euphytica 66, 135-144.

Blank, R., G. Campbell, A. Calabro and P. D'Eustachio, 1988: A linkage map of chromosome 12: localization of $I g h$ and effects of sex and interference on recombination. Genetics 120, 10731083.

Budin, K., 1969: Derivation of potato haploids. Genetika 5, 43-50.

Bugarova, Z. and A. Pretova, 1996: Isolated microspore cultures in Solamum tuberosum cultivars. Biologia, Bratislava 51, 411-416.

Caligari, P. D. S., W. Powell, K. Liddell, M. J. de Maine and G. E. L. Swan, 1988: Methods and strategies for detecting Solanum tuberosum dihaploids in interspecific crosses with S. phureja. Ann. Appl. Biol. 112, 323-328.

Calleberg, E., S. Ingileif, S. Kristjansdottir and L. Johansson, 1989: Anther cultures of tetraploid Solanum genotypes - the influence of gelling agents and correlations between incubation temperature and pollen germination temperature. Plant Cell Tiss. Org. Cult. 19,189-197.

Calleberg, E. and L. Johnson, 1993: The effect of starch and incubation temperature in anther culture of potato. Plant Cell Tiss. and Org. Cult. 32, 27-34. 
Cappadocia, M and M. Ahmim, 1988: Comparison of two culture methods for the production of haploids by anther culture in Solamum chacoense. Can. J. Bot. 66, 1003-1005.

Cappadocia, M., D. Cheng and R. Ludlum-Simonette, 1984: Plant regeneration from in vitro culture of anthers of Solamum chacoense Bitt. and interspecific diploid hybrids S. tuberosum $\mathrm{L} \times$ S. chacoense Bitt. Theor. Appl. Genet. 69, 139-143.

Chase, S. S., 1963: Analytic breeding in Solanum tuberosum L.- a scheme utilizing parthenotes and other diploid stocks. Can J. Genet. Cytol. 5, 359-363.

Clulow, S. A., M. J. Wilkinson and L. R. Burch, 1993: Solanum phureja genes are expressed in the leaves and tubers of aneusomatic potato dihaploids. Euphytica. 69, 1-6.

Clulow, S. A., M. J. Wilkinson, R. Waugh, E. Baird, M. J. De Maine and W. Powell, 1991:

Cytological and molecular observations on Solamum phureja-induced dihaploid potatoes. Theor. Appl. Genet. 82, 545-551.

Daly, D. C., 1996: The leaf that launched a thousand ships. Nat. Hist. 24-32.

De Paepe, R., D. Prat and T. Huguet, 1982/83: Heritable nuclear DNA changes in doubled haploid plants obtained by pollen culture of Nicotiana sylvestris. Plant Sci. 28, 11-28.

Devaux, P., 1988. Comparison of anther culture and the Hordeum bulbosum method for the production of doubled haploids in winter barley. II. Variation of chromosome number and seed set in the progeny. Plant Breed. 100, 181-187.

Devaux, P., A. Kilian and A. Kleinhofs, 1995: Comparative mapping of the barley genome with male and female recombination-derived, doubled haploid populations. Mol. Gen. Genet. 249, $600-608$.

Dhillon, S., E. Wernsman and J. Miksche, 1983: Evaluation of nuclear DNA content and heterochromatin changes in anther-derived dihaploids of tobacco (Nicotiana tabacum) cv. Coker 139. Can. J. Genet. Cytol. 25, 169-173.

Dib, C., S. Faure, C. Fizames, D. Samson, N. Drouot, A. Vignal, P. Millasseau, S. Marc, J. Hazan, E. Seboun, M. Lathrop, G. Gyapay, J. Morissette and J. Weissenbach, 1996: A comprehensive genetic map of the human genome based on 5, 264 microsatellites. Nature 380, $152-154$.

Dunwell, J. and N. Sunderland, 1973: Anther culture of Solanum tuberosum L. Euphytica. 22, 317-323. 
Ellegren, H., B. Chowdhary, M. Fredholm, B. Hoyheim, M. Johansson, P. Nielsen, P. Thomsen and L. Andersson, 1994: A physically anchored linkage map of pig chromosome 1 uncovers sexand position-specific recombination rates. Genomics 24, 342-350.

Evans, D., W. Sharp and H. Medina-Filho, 1984: Somaclonal and gametoclonal variation. Am. J. Bot. 71, 759-774.

Foisset, N. and R. Delourme, 1996: Segregation distortion in androgenic plants. In: Mohan Jain, S., S. K. Sopory and R. E. Veilleux, eds. In Vitro Haploid Production in Higher Plants, Vol.3. Kluwer Academic Publishers, Dordrecht, The Netherlands, 189-201.

Foroughi-Wehr, B., H. Wilson, G. Mix, and H. Gaul, 1977: Monohaploid plants from anthers of a dihaploid genotype of Solanum tuberosum L. Euphytica. 26, 361-367.

Frandsen, N., 1968: Die Plastidenzahl als Merkmal bei der Kartoffel. Theor. Appl. Genet. 38, 153-167.

Fregene, M., F. Angel, R. Gomez, F. Rodriguez, P. Chavarriaga, W. Roca, J. Tohme and M. Bonierbale, 1997: A molecular genetic map of cassava (Manihot esculenta Crantz). Theor. App1. Genet. 95, 431-441.

Hawkes, J. G. 1990: The Potato: Evolution, Biodiversity, and Genetic Resources. Smithsonian Institution Press; Washington, D. C.

Hermsen, J. G. T. and J. Verdenius, 1973: Selection from Solamum tuberosum Group Phureja of genotypes combining high-frequency haploid induction with homozygosity for embryo-spot. Euphytica 22, 244-259.

Hougas, R. and S. Peloquin, 1957: A haploid plant of the potato variety Katahdin. Nature 180, 1209-1210.

Hougas, R., S. Peloquin and A. Gabert, 1964: Effect of seed-parent and pollinator on frequency of haploids in Solanum tuberosum. Crop Sci. 4, 593-595.

Hougas, R., S. Peloquin and R. Ross, 1958: Haploids of the common potato. J. Hered. 47, 103107.

Irikura, Y., 1975: Induction of haploid plants by anther culture in tuber-bearing species and interspecific hybrids of Solamum. Potato Res. 18, 133-140.

Irikura, Y. and S. Sakaguchi, 1972: Induction of 12-chromosome plants from anther culture in a tuberous Solanum. Potato Res. 15, 170-173. 
Ivanovskaja, E., 1939: Haploid plant of Solamum tuberosum. Acad. Sci. U.R.S.S. 24, 517.

Jacobsen, E. and M. Ramanna, 1994: Production of monohaploids of Solamum tuberosum and their use in genetics, molecular biology and breeding. In: Bradshaw, J. and G. MacKay, eds. Potato Genetics. CAB Int.: Oxon, 155-170.

Jacobsen, E. and S. Sopory, 1978: The influence and possible recombination of genotypes on the production of microspore embryoids in anther cultures of Solamum tuberosum and dihaploid hybrids. Theor. Appl. Genet. 52, 119-123.

Jansky, S. H., G. L. Yerk and S. J. Peloquin, 1990: The use of potato haploids to put $2 x$ wild species germplasm into a usable form. Plant Breed. 104, 290-294.

Johansson, L., 1983: Embryogenesis in anther cultures of some species in the families Solanaceae, Ranunculaceae and Papaveraceae. Abstracts of Uppsala Dissertations from the Faculty of Sciences. Upsala, Sweden.

Johansson, L., 1986: Improved methods for induction of embryogenesis in anther cultures of Solamum tuberosum. Potato Res. 29, 179-190.

Johansson, L., 1988: Increased induction of embryogenesis and regeneration in anther cultures of Solanum tuberosum L. Potato Res. 31, 145-149.

Kreike, C. and W. Stiekema, 1997: Reduced recombination and distorted segregation in a Solanum tuberosum $(2 x) \times S$. spegazzinii $(2 x)$ hybrid. Genome $40,180-187$.

Kumashiro, T. and T. Oinuma, 1985: Comparison of genetic variability among anther-derived and ovule-derived doubled haploid lines of tobacco. Jpn. J. Breed. 35, 301-310.

Lamm, R., 1938: Notes on a haploid potato hybrid. Hereditas 24, 391.

Lagercrantz, U. and D. Lydiate, 1995: RFLP mapping in Brassica nigra indicates differing recombination rates in male and female meioses. Genome 38, 255-264.

Liu, C. and D. S. Douches, 1993: Production of haploids of potato (Solamum tuberosum subsp. tuberosum) and their identification with electrophoretic analysis. Euphytica. 70, 113-126.

Logue, S., 1996, Genetic stability in microspore-derived doubled haploids. In: Mohan Jain, S., S. K. Sopory and R. E. Veilleux, eds. In Vitro Haploid Production in Higher Plants, Vol.3. Kluwer Academic Publishers, Dordrecht, The Netherlands, 1-51. 
Marklund, L., M. Moller, B. Hoyheim, W. Davies, M. Fredholm, R. Juneja, P. Mariani, W. Coppieters, H. Ellegren and L. Andersson, 1996: A comprehensive linkage map of the pig based on a wild pig-Large White intercross. Anim. Genet. 27, 255-269.

Meyer, R., F. Salamini and H. Uhrig, 1992: Biotechnology and plant breeding: relevance of cell genetics in potato improvement. Proc. Royal Soc. Edinburgh 99B (3/4), 11-21.

Montelongo-Escobedo, H. and P. R. Rowe, 1969: Haploid induction in potato: cytological basis for the pollinator effect. Euphytica. 18, 116-123.

Murigneux, A., S. Baud, M. Beckert, 1993: Molecular and morphological evaluation of doubledhaploid lines in maize. 2. Comparison with single-seed-descent lines. Theor. Appl. Genet. 87, 278-287.

Nielsen, M. T. and G. B. Collins, 1989: Variation among androgenic and gynogenic doubled haploids of tobacco (Nicotiana tabacum L.) Euphytica 43, 263-267.

Owen, H., R. Veilleux, F. Haynes and K. Haynes, 1988: Photoperiod effects on $2 n$ pollen production, response to anther culture, and net photosynthesis of a diplandrous clone of Solanum phureja. Am. Potato J. 131-139.

Pehu, E., 1986: Analysis of anther-derived plants of Solamum phureja: variation in ploidy, photosynthetic efficiency and structure of the nuclear genome. Ph.D. dissertation, Virginia Polytechnic Institute and State University, Blacksburg, Virginia.

Peloquin, S. J. and R. W. Hougas, 1959: Decapitation and genetic markers as related to haploidy in Solanum tuberosum. Eur. Potato J. 2, 176-183.

Powell, W., P. Caligari, W. Thomas, 1986: Comparison of spring barley lines produced by single seed descent, pedigree inbreeding, and doubled haploidy. Plant Breed. 97, 138-146.

Reynolds, T., 1990: Interactions between calcium and auxin during pollen androgenesis in anther cultures of Solanum carolinense L. Plant Sci. 72, 109-114.

Rivard, S., M. Cappadocia, and B. Landry, 1996: A comparison of RFLP maps based on anther culture derived, selfed, and hybrid progenies of Solanum chacoense. Genome. 39, 611-621.

Rogers, J., S. Witte, C. Kammerer, J. Hixson, and J. MacCluer, 1995: Linkage mapping in Papio baboons: conservation of a syntenic group of six markers on human chromosome 1 . Genomics $28,251-154$.

Rohrer, G., L. Alexander, C. Beattie, P. Wilkie, G. Flickinger, L. Schook, A. Paszek, L. Andersson, P. Mariani, L. Marklund, M. Fredholm, B. Hoyheim, A. Archibald, V. Nielsen, D. 
Milan and M. Groenen, 1997: A consensus linkage map for swine chromosome 7. Anim. Genet. $28,223-229$.

Rokka, V., L. Pietila and E. Pehu, 1996: Enhanced production of dihaploid lines via anther culture of tetraploid potato (Solanum tuberosum L. ssp. tuberosum). Am. Potato J. 73, 1-12.

Simon, P. and S. Peloquin, 1977: The influence of paternal species on the origin of callus in anther culture of Solanum hybrids. Theor. Appl. Genet. 50, 53-56.

Singsit, C. and R. Hanneman, Jr., 1987: Haploids of tetraploid ( $2 n=4 x=48)$ Mexican potato species - their extraction, cytology and crossability. Am. Potato J. 64, 469-482.

Singsit, C. and R. E. Veilleux, 1988: Intra- and interspecific transmission of androgenetic competence in diploid potato species. Euphytica 43, 105-112.

Sonnino, A., S. Tanaka, M. Iwanaga and L. Schilde-Rentschler, 1989: Genetic control of embryo formation in anther culture of diploid potatoes. Plant Cell Rep. 8, 105-107.

Sopory, S. and P. Rogan, 1976: Induction of pollen divisions and embryoid formation in anther cultures of some dihaploid clones of Solanum tuberosum. Z. Pflanzenphysiol. 80, 77-80.

Sopory, S., 1977: Differentiation in callus from cultured anthers of dihaploid clones of Solamim tuberosum. Z. Pflanzenphysiol. 82, 88-91.

Sopory, S., 1979: Effect of sucrose, hormones, and metabolic inhibitors on the development of pollen embryoids in anther cultures of dihaploid Solanum tuberosum. Can. J. Bot. 57, 26912694.

Sopory, S. and B. Tan, 1979: Regeneration and cytological studies of anther and pollen calli of dihaploid Solamum tuberosum. Z. Pflanzenzuchtg. 82, 31-35.

Sopory, S., E. Jacobsen, and G. Wenzel, 1978: Production of monohaploid embryoids and plantlets in cultured anthers of Solanum tuberosum. Plant Sci. 12, 47-54.

Tanhuanpaa, P., J. Vilkki and h. Vilkki, 1994: Segregation and linkage analysis of DNA markers in microspore derived and $\mathrm{F}_{2}$ populations of oilseed rape (Brassica napus L.). Euphytica 74, 5965 .

Tanzi, R., P. Watkins, G. Stewart, N. Wexler, J. Gusella and J. Haines, 1992: A genetic linkage map of human chromosome 21: Analysis of recombination as a function of sex and age. Am. J. Hum. Genet. 50, 551-558. 
Taylor, T. and R. Veilleux, 1992: Inheritance of competencies for leaf disc regeneration, anther culture, and protoplast culture in Solamum phureja and correlations among them. Plant Cell Tiss. Org. Cult. 31, 95-103.

Tiainen, T., 1992a: The role of ethylene and reducing agents on anther culture response of tetraploid potato (Solanum tuberosum L.). Plant Cell Rep. 10, 604-607.

Tiainen, T., 1992b: The influence of culture conditions on anther culture response of commercial varieties of Solamum tuberosum L. Plant Cell Tiss. Org. Cult. 30, 211-219.

Tiainen, T., 1993: The influence of hormones on anther culture response of tetraploid potato (Solamum tuberosum L.). Plant Sci. 88, 83-90.

Uhrig, H., 1985: Genetic selection and liquid medium conditions improve the yield of androgenetic plants from diploid potatoes. Theor. Appl. Genet. 71, 455-460.

Uhrig, H., 1983: Breeding for Globodera pallida resistance in potatoes. Z. Pflanzenzuchtg. 91, 211-218.

Uhrig, H. and F. Salamini, 1987: Dihaploid plant production from $4 x$-genotypes of potato by the use of efficient anther plants producing tetraploid strains (4x EAPP-clones) - Proposal of a breeding methodology. Plant Breed. 98, 228-235.

Uijtewaal, B. A., D. J. Huigen and J. G. Th. Hermsen, 1987: Production of potato monohaploids $(2 n=x=12)$ through prickle pollination. Theor. Appl. Genet. 73, 751-758.

van Bruekelen, E. W. M., M. S. Ramanna and J. G. Th. Hermsen, 1975: Monohaploids ( $n=x=12$ ) from autotetraploid Solamum tuberosum $(2 n=4 x=48)$ through two successive cycles of female parthenogenesis. Euphytica. 24, 567-574.

van Breukelen, E. W. M., 1981: Pseudogamic production of dihaploids and monoploids in Solamum tuberosum and some related species. Agricultural Research Reports 908. Center for Agricultural Publishing and Documentation, Wageningen.

Veilleux, R. E., 1996: Haploidy in important crop plants - potato. In: Mohan Jain, S., S. K. Sopory and R. E. Veilleux, eds. In vitro Haploid Production in Higher Plants, Vol. 3. Kluwer Academic Publishers: Dordrecht, The Netherlands, 37-49.

Veilleux, R., J. Booze-Daniels and E. Pehu, 1985: Anther culture of a $2 n$ pollen producing clone of Solanum phureja Juz. and Buk. Can. J. Genet. Cytol. 27, 559-564.

Veilleux, R. and A. Johnson, 1998: Somaclonal variation: molecular analysis, transformation interaction, and utilization. Plant Breed. Rev. In press. 
Wagenheim, K., S. Peloquin and R. Hougas, 1960: Embryological investigations on the formation of haploids in the potato (Solamum tuberosum). Plant Breed. 91, 391-399.

Wang, G., V. Hyne, S. Chao, Y. Henry, J. De Buyser, M. Gale and J. Snape, 1995: A comparison of male and female recombination frequency in wheat using RFLP maps of homoeologous group 6 and 7 chromosomes. Theor. Appl. Genet. 91, 744-746.

Wenzel, G., O. Scheider, T. Przewozny, S. K. Sopory and G. Melchers, 1979: Comparison of single cell culture derived Solanum tuberosum L. plants and a model for their application in breeding programs. Theor. Appl. Genet. 55, 49-55.

Wenzel, G. and H. Uhrig, 1981: Breeding for nematode and virus-resistance in potato via anther culture. Theor. Appl. Genet. 59, 333-340.

Wilkinson, M. J., S. T. Bennett, S. A. Clulow, J. Allainguillaume, K. Harding and M. D. Bennett, 1995: Evidence for somatic translocation during potato dihaploid induction. Heredity 74, 146151.

Xu, Y., L. Zhu, J. Xiao, N. Huang and S. McCouch, 1997: Chromosomal regions associated with segregation distortion of molecular markers in $\mathrm{F}_{2}$, backcross, doubled haploid, and recombinant inbred populations in rice (Oryza sativa L.). Mol. Gen. Genet. 253, 535-545.

Zivy, M., P. Devaux, J. Blaisonneau, R. Jean and H. Thiellement, 1992: Segregation distortion and linkage studies in microspore-derived double haploid lines of Hordeum vulgare $\mathrm{L}$. Theor. Appl. Genet. 83, 919-924. 